\title{
GIAMBATTISTA VICO Y LA POLÍTICA DE LA RAZÓN
}

\section{Pierre Girard \\ (Universidad de Lyon)}

RESUMEN : Este artículo pretende trazar la genealogía de una interpretación de Vico nacida de la redefinición de la razón y del estatus político de su pensamiento.

Palabras Clave : Vico, $350^{\circ}$ Aniversario, razón, política, práctica, Descartes, materialismo, libertas philosophan$d i$, N. Badaloni, P. Girard.

\section{Giambattista Vico and the politics of reason}

ABSTRACT: This article aims to trace the genealogy of an interpretation of Vico, born of the redefinition of reason and the political status of his thought.

KEYWORDS: Vico, $350^{\text {th }}$ Anniversary, reason, politics, practice, Descartes, materialism, libertas philosophandi, N. Badaloni, P. Girard.

\section{Giambattista Vico e la politica della ragione}

RIASSUNTO: Questo articolo intende tracciare la genealogia di un'interpretazione di Vico nata dalla ridefinizione della ragione e dallo statuto politico del suo pensiero.

PAROLE ChIAVE: Vico, $350^{\circ}$ Anniversario, ragione, politica, pratica, Descartes, materialismo, libertas philosophan$d i$, N. Badaloni, P. Girard.

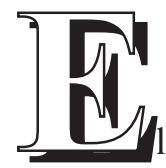

proyecto de considerar retrospectivamente la interpretación que se tiene de un autor al que se ha frecuentado durante muchos años, y gracias al cual se ha podido viajar, intercambiar, entablar amistades profundas y mantener estimulantes discusiones intelectuales, es siempre un ejercicio peligroso. El peligro es doble. El primero es el que acecha al especialista encerrado en una lectura fijada hace ya mucho tiempo y que tiende a congelar su aproximación al autor en torno a algunas tesis principales. El segundo, que es quizás mayor, consiste en proponer una lectura retrospectiva, animada por el "senno di poi" que tiende a exponer de manera lineal y sin la menor asperidad las distintas etapas que han llevado a esa interpre- 
tación. En este sentido, hablar de $m i$ Vico me obliga a esforzarme por aplicar aquello que el filósofo napolitano exigía a una ciencia verdaderamente "nueva": ser capaz de evitar la «boria de' dotti», ${ }^{1}$ esa inclinación que nos lleva a exportar nuestras categorías y nuestros métodos a un objeto que les es heterogéneo. Querría pues, en las líneas que siguen, escapar a esta tentación, evitar proponer una interpretación estática y congelada de Vico, la interpretación a la que yo he podido llegar, para, al contrario, ofrecer el relato de un recorrido junto a un autor fascinante, cuya riqueza consiste precisamente en poder alimentar la reflexión y la evolución de esta sin dejarse encerrar en una lectura inmóvil y unilateral.

\section{ENCUENTROS}

El relato de este recorrido es también el de una serie de encuentros. Primero, con el autor y con sus textos, pero también con aquellos que supieron hacerlo llegar al joven estudiante francés que realizaba una estancia de intercambio en la Scuola Normale Superiore de Pisa a mediados de los años '90. Quiero subrayar la importancia de estos encuentros sin los cuales $m i$ Vico nunca hubiera sido "mío" en el sentido propio de la palabra. Tuve, en efecto, la suerte de conocer, especialmente en Italia, a numerosos profesores que siempre me abrieron sus puertas y me escucharon sin imponerme nunca una lectura, dejándome así la posibilidad de construir la mía: Paolo Cristofolini, al que me une una amistad que ya dura más de veinte años; Fulvio Tessitore, que codirigió con Alain Pons mi Máster de filosofía, y el conjunto de los miembros del "Centro di Studi Vichiani", con Manuela Sanna y Giuseppe Cacciatore a la cabeza. Sin ellos, yo nunca habría podido ni tan siquiera plantearme cuál era $m i$ Vico. Estos agradecimientos no son en absoluto un mero trámite sino el reconocimiento objetivo del trabajo incansable y admirable de estos investigadores, una labor a la que debo la construcción de $m i$ Vico. $^{2}$

Entre estos encuentros, hay también libros y críticos que me marcaron. También aquí la lista es larga y va de Pietro Piovani a Paolo Cristofolini, pasando por Giuseppe Galasso, Andrea Battistini, Riccardo Caporali, Massimo Lollini, Jürgen Trabant o Enrico Nuzzo. La lectura de la monografía de Croce, de 1911, fue desde luego una lectura importantísima que a mi entender planteaba a la perfección los problemas que emergen del pensamiento de Vico sin llegar nunca a interpretarlos adecuadamente. Croce discernía admirablemente cuáles eran las dificultades de la empresa de Vico, pero las interpretaba cada vez como un fallo en la empresa filosófica cuando, a mi juicio el gran interés del filósofo napolitano reside en el

1. En realidad, Vico distingue dos tipos de «boria»: la de los «sabios» y la de las «naciones». Véase $S N 44$, § 122128.

2. Como muestra de este trabajo, remitimos al magnífico "Portale Vico" (http://www.giambattistavico.it) que constituye una indispensable herramienta de trabajo para toda la comunidad de investigadores que se dedican al pensamiento de Vico. 
hecho mismo de ser capaz de plantear esas dificultades. Entre estas lecturas críticas, una fue, y sigue siendo, especialmente decisiva para mí: la de la monografía de Nicola Badaloni publicada en 1961. ${ }^{3}$ Dejando de lado la magistral erudición de esa obra, Badaloni enmarcaba a Vico en el Seicento napolitano y en la complejidad de la libertas philosophandi de los novatores del ceto civile napolitano, y ofrecía una interpretación totalmente nueva de Vico como pensador de la Modernidad. Esta perspectiva me permitió dejar atrás el angosto horizonte de la lectura de Croce. Debo, sin embargo, reconocer que aunque Nicola Badaloni haya constituido una etapa decisiva en la construcción de $m i$ Vico, no por ello hago mías todas sus posiciones. Sí hago mío sin ambages el dinamismo de su lectura innovadora que abre un espacio para otras lecturas. Este fue también el caso de mi lectura de Ernesto Grassi, ${ }^{4}$ que me fascinó y desorientó a la vez. La construcción de $m i$ Vico debe mucho al cuestionamiento al que someto las tesis de Grassi, especialmente en lo que al lugar que ocupa la razón se refiere. ${ }^{5}$

\section{LENGUA Y CORPUS}

El relato de mi encuentro con Vico pasa también por rendir cuentas de una lectura que me desarmó, una lectura en cierto modo descorazonadora para un estudiante francés de filosofía acostumbrado al poder absoluto de la tradición cartesiana, de un verbo que se supone - sin duda con demasiada facilidad- "claro y distinto". La lectura de Vico supone una dificultad que no debe ser silenciada, sino interpretada y comentada. Nunca he considerado que esta dificultad fuera algo que tuviera que subsanar, buscando aquí y allá resúmenes y esquemas explicativos que presentaran el pensamiento de un Vico desencarnado, privado así de su lengua. La especificidad de esta lengua es de sobra conocida y son muchos los estudios que han sabido mostrar sus elementos, desde Mario Fubini ${ }^{6}$ hasta Andrea Battistini. ${ }^{7}$ Como

3. N. Badaloni, Introduzione a G. B. Vico, Feltrinelli, Milán, 1961. Véase también sus introducciones a las Opere filosofiche (1971) y a las Opere giuridiche (1974) de Vico (Sansoni, Florencia). Sobre la interpretación de N. Badaloni, véase el estudio de S. LANDUCCI, «Vico e il previchismo nella nuova interpretazione di Nicola Badaloni», Belfagor, XVI, 1961, pp. 347-361. También puede consultarse el gran trabajo de R. CAPORALI, «Ragione e natura nella filosofia di Vico. La lettura di Nicola Badaloni», Bollettino del Centro di studi vichiani, XII-XIII, 1982-1983, pp. 151-197.

4. Véase en especial Vico e l'umanesimo, Guerini e Associati, Milán, 1992. Para una presentación sintética de la lectura de Grassi, véase A. Pons, «Ernesto Grassi lecteur de Vico», en Présence de Vico, Actes du colloque "Giambattista Vico aujourd'hui», dir. R. PINERI, Main d'œuvre, Montpellier, 1996, pp. 13-29. Véase el magnífico estudio de M. MARASSI, «Ernesto Grassi y el problema de la metáfora en el De nostri temporis studiorum ratione», Cuadernos sobre Vico, 9-10, 1998, pp. 89-108. [Cfr. de ambos dos autores sobre Grassi: Cuadernos sobre Vico, 1314, 2001-2002, pp. 47-53 (Pons) y pp. 55-69 (Marassi). N.E.]

5. Véase P. GIRARD, Giambattista Vico. Rationalité et politique. Une lecture de la Scienza nuova, PUPS, París, 2008, pp. 101 y ss.

6. Sobre este papel sugestivo de la escritura y el estilo de Vico, véanse las observaciones de M. FuBINI, «Umanesimo e umanità nell'opera di G. B. Vico», en Stile e umanità di G. B. Vico, Riccardo Ricciardi Ed., Milán-Nápoles, 1945, pp. 182 y ss.

7. La degnità della retorica. Studi su G. B. Vico, Pacini Editore, Pisa, 1975.

Cuadernos sobre Vico 32 (2018) 
estos investigadores, también yo estoy convencido de que la especificidad de esta lengua no es la de un envoltorio formal del que se podría prescindir, como si la filosofía de Vico se pudiera disociar de la lengua en la que se encarna. ${ }^{8}$ No es posible acceder al pensamiento de Vico sino a través de una lengua única, oscura y a la vez sublime, una lengua viva que convierte a la Scienza nuova en particular en un verdadero laboratorio linguístico. Esta lengua tiene un sentido filosófico preciso en la medida en que es el instrumento forjado por el propio Vico ${ }^{9}$ para penetrar en el oscuro mundo de las naciones y para evitar la «boria de' dotti».

Enfrentarse a Vico es también enfrentarse a un corpus diverso, compuesto por una multiplicidad de textos de niveles retóricos muy diversos. Esta diversidad es de sobras conocida, pero pocos son los estudios que se posicionan explícitamente sobre ella. En lo que a mí respecta, siempre he intentado leer a Vico de manera global sin perder de vista el conjunto de su producción, ya sea filosófica, jurídica, retórica, epistolar, etc. Pero esta manera de afrontar la obra en su totalidad implicaba, en mi opinión, ser capaz de establecer una jerarquía entre los diferentes elementos que la componen. Siempre he considerado que extraer del corpus tal o cual obra para estudiarla aparte y convertirla en la clave de la comprensión de la filosofía de Vico era muy discutible, a pesar de la gran calidad de muchas interpretaciones así elaboradas. ${ }^{10}$

En este aspecto, he asumido las indicaciones del propio Vico, que no duda en atribuirle a su Scienza nuova el lugar a posteriori dominante, central, en el conjunto de su obra. ${ }^{11}$ Esto conlleva varias consecuencias: la primera consiste en pensar la articulación entre la Scienza nuova y los otros textos. La segunda consiste en no considerar la Scienza nuova como un texto único, sino como una producción en movimiento, un work in progress, como lo escribe con gran acierto Paolo Cristofolini, ${ }^{12}$ y, por tanto, considerar a la vez las tres versiones de la Scienza nuova y las diferentes modificaciones y añadidos entre los textos. He de reconocer que al prestar así atención a la dinámica de la Scienza nuova, la versión de 1725 me ha parecido a menudo la más clara y la más decisiva en muchos aspectos con respecto a la historia de la filosofía. Esta voluntad de jerarquizar me llevó también a tomar una posición crítica frente al De antiquissima Italorum sapientia, del que siempre he pensado que constituía una etapa desde luego importante en el pensamiento de Vico, pero una mera etapa algunas de cuyas tesis Vico abandonaría años más

8. Véase B. De Giovanni, «Vico baroque», Critique, 452-453, enero-febrero 1985, pp. 163-180.

9. Véase J. Trabant, «La lingua di questa Scienza: lingua antica-scienza nuova», en Pensar para el nuevo siglo. Giambattista Vico y la cultura europea, E. Hidalgo-Serna, M. Marassi, J.M. SeVIlla, J. Villalobos (Eds.), La Città del Sole, Nápoles, 2001, vol. I, pp. 189-218.

10. Sobre este punto, me permito reenviar a mi estudio «Vico en France: quelques interprétations critiques», Revue des études italiennes, L'Âge d'homme, París, 2007, pp. 9-22.

11. Véase P. GIRARD, «Science et sagesse poétique dans la philosophie de Giambattista Vico», en La Scienza nuova: quelle science? Le conflit des interprétations, bajo la dirección de A. Tosel, Niza, Noesis, 8, 2005, pp. 125-139. 12. Véase P. CRistofolini, Vico et l'histoire, PUF, París, 1995, p. 15. 
tarde. ${ }^{13}$ Siempre me he resistido a sacar este texto del corpus y a considerarlo como una clave interpretativa general para forjar una lectura 'metafísica' de Vico.

\section{MODERNIDAD, RAZÓN Y POLÍTICA}

Además del camino intelectual que acabo de esbozar en las líneas que preceden, creo que $m i$ Vico no consiste tanto en una suma de tesis o de afirmaciones como en una serie de trayectos intelectuales, de intercambios y en una constante interrogación suscitada por un pensamiento cuya fuerza reside a mi juicio no tanto en su capacidad para reducirse a un puñado de conceptos sino en ser capaz de abrir un fecundo campo de investigación. Dicho esto, siempre es posible reducir una interpretación a unas cuantas ideas principales. Vico siempre me ha parecido ser uno de los pensadores más originales de la modernidad europea, capaz de anticipar las grandes problemáticas de nuestro mundo actual. ${ }^{14}$ Del mismo modo, nunca he pensado que, por muy fascinante que sea la interpretación viquiana de la imaginación y de los productos de la «fantasia», Vico intentase sustituir la razón por la imaginación. En efecto, a mi juicio Vico no pierde nunca de vista la razón, que constituye la finalidad última y claramente reivindicada de su pensamiento: en vez de apartar la instancia racional, la obra de Vico busca al contrario llevarla al límite. ${ }^{15}$ La relación con Descartes es decisiva en este aspecto. Siempre he considerado que la relación que Vico mantiene con Descartes -y yo diría más particularmente con la tradición cartesiana- es compleja y no puede en absoluto reducirse a una mera oposición. He tratado esta cuestión en diversas ocasiones. ${ }^{16}$ A mi entender, más que oponerse a Descartes, Vico considera que la empresa cartesiana se ha detenido prematuramente y lamenta que se limite al escueto marco de lo "claro" y lo "distinto". Si no queremos limitarnos a «un mundo de hombres compuesto de líneas, de números y de signos algebraicos», ${ }^{17}$ si se quiere afrontar el mundo real, con su compleji-

13. Sobre este punto, remito a mi estudio: Giambattista Vico. Rationalité et politique. Une lecture de la Scienza nuova, op. cit., pp. 12 y ss.

14. Véase P. GIRARD, «Modernità e 'ragione tutta spiegata' in Vico», en Razionalità e modernità in Vico, M. VANZuLLI (Dir.), Mimesis, Milán, 2012, pp. 245-261.

15. Véase P. GIRARD, «Las condiciones y los límites de la racionalidad en la Scienza nuova. Las metamorfosis de la razón», Cuadernos sobre Vico, 13-14, 2001-2002, pp. 127-138.

16. Véase en particular «Giambattista Vico critique de Descartes ?», en Qu'est-ce qu'être cartésien ?, D. KolESNIKANTOINE (Dir.), ENS Éditions, Lyon, 2013, pp. 503-519; «Les usages de Malebranche dans la réception du cartésianisme à Naples», en Les Malebranchismes des Lumières. Études sur les réceptions contrastées de la philosophie de Malebranche, fin XVIIe et XVIIIe siècles, D. KolESNIK-ANTOINE (Dir.), Honoré Champion, París, 2014, pp. 225246; "Vico e a tradição cartesiana», en Metafisica do Gênero Humano: naturaleza e história na obra de Giambattista Vico, F. Lomonaco, H. Guido, S. De Amorim e Silva Neto (Dir.), EDUFU, Uberlândia, 2018, pp. 255-284; "The Materialist Reception of the Cartesian Physics in Naples», en Physics and Metaphysics in Descartes and his Reception, ed. de D. Antolne Mahut y S. Roux, Routledge, Nueva York-Londres [en prensa, 2019], pp. 175-187.

17. Carta a Francesco Saverio Estevan, 12 de enero 1729, en Opere, a cargo de A. BATTISTINI, Mondadori, Milán, 1990, p. 335 («Allo incontro, come se i giovani dalle accademie dovesser uscire nel mondo degli uomini, il qual fossesi composto di linee, di numeri e di spezie algebraiche $[\ldots] »)$. 
dad, con su envergadura política e histórica, hay que evitar la «ficción cartesiana» («Non fingerassi qui ciò che astutamente finse Renato Delle Carte [... $] »),{ }^{18}$ la mera evidencia, y ser capaces de penetrar, con las debidas precauciones, en el mundo de los «auttori delle nazioni». Este aspecto queda, a mi juicio, explícitamente puesto en evidencia en el «grabado colocado en el frontispicio» que «sirve de introducción a la obra». ${ }^{19}$ En él, Vico muestra que toda la originalidad de su empresa consiste precisamente en no conformarse con la mirada diáfana entre la providencia y la metafísica, sino en seguir cuidadosamente la refracción del rayo y en esforzarse por pensar las condiciones de posibilidad de una «metafísica poética».20

La relación con Descartes es a mi entender muy sintomática de la estrategia del sistema de referencias en la Scienza nuova y de la capacidad evidente de Vico para leer a otros filósofos, no tanto para inspirarse directamente de ellos o para criticarlos frontalmente, sino para integrarlos en su propio pensamiento, dándoles un valor y una función nuevos. Es el caso, de manera evidente, de Descartes, pero también de Spinoza y de la tradición materialista y, en primer lugar, la de Lucrecio, ${ }^{21}$ cuyo pensamiento es central en la economía de la Scienza nuova, como lo recalcó Enzo Paci y su estudio pionero. ${ }^{22}$

De manera general, diría que mi interpretación se ha construido en dos etapas. La primera, una vez finalizada mi aproximación a la totalidad del corpus, consistió en proponer una lectura global de la Scienza nuova. Pretendía, ante todo, resistir a la tentación de resumir este texto para ser capaz de entenderlo en toda su complejidad, especialmente en su complejidad formal, como lo he indicado más arriba. Se trataba para mí de no caer en la poderosa tentación típica de la historiografía filosófica francesa, ilustrada por ejemplo por los estudios admirables de Martial Gueroult sobre la filosofía moderna, que consiste en evaluar una filosofía en función de su carácter sistemático. Desde luego, Vico recuerda que su Scienza nuova es un "sistema", pero me parecía que cabía entenderlo no como un pensamiento que pudiera exponerse de manera sincrónica, sino como un sistema en movimiento, abierto. Desde esta perspectiva, la Scienza nuova me ha parecido siempre un laboratorio abierto, que distingue las etapas de proposición de las de experimentación, de las de conclusión, y en ningún caso una mera exposición retórica estática. ${ }^{23}$

La segunda etapa que puedo discernir en mi Vico es la del contexto. También en este aspecto debía resistir a otra poderosa tentación propia de la historiografía

18. Vita, en Opere, op. cit., p. 7.

19. $S N 44$, «Idea dell'opera», $\S \S 1-42$.

20. Sobre este punto, véase nuestro estudio «Homo non intelligendo fit omnia. Vico et la métaphysique poétique», en Les métaphysiques des Lumières, P. Girard, C. Leduc, Mitia RiouX-Beaulne (Dir.), Classiques Garnier, París, 2016, pp. 35-54.

21. Véase P. GIRARD, «Echi lucreziani nel pensiero vichiano», en Lucrezio e la modernità. I secoli XV-XVII, a cargo de F. Del Lucchese, V. Morfino, G. Mormino, Bibliopolis, Nápoles, 2011, pp. 253-270.

22. Ingens Sylva, Bompiani, Milán, 1994 [1949].

23. Es el objeto de mi Giambattista Vico. Rationalité et politique. Une lecture de la Scienza nuova, op. cit., passim. 
filosófica francesa -aunque hoy ya se considere en gran parte caduca- que consistía en comparar los sistemas filosóficos como mónadas que se enfrentan en un mundo de conceptos puros en el que la historia, la política, las relaciones sociales y la retórica estaban totalmente ausentes. El reconocimiento de la originalidad de la sistematicidad del pensamiento de Vico no significaba, pues, en ningún modo aceptar un pensamiento desgajado de sus condiciones de producción. Como varios de mis compañeros viquianos, nunca he considerado que Vico fuera un pensador aislado en su época, ni tampoco un "arcaico". Este esquema es una construcción decimonónica. La compleja relación que mantiene Vico con la historia de la filosofía muestra al contrario hasta qué punto su pensamiento se nutre de la tradición y de la historia de las ideas. No hacer de Vico un pensador aislado, una "pirámide en el desierto", ${ }^{24}$ suponía también ponerlo en relación con su contexto y en particular con la segunda mitad del Seicento napolitano, fascinante en muchos aspectos. También en este aspecto le debo mucho a la investigación pionera y magistral de Nicola Badaloni. Pero tenía que ir más allá y pensar la articulación entre Vico y la tradición investigante, los grandes novatores, de Tommaso Cornelio a Leonardo Di Capua, de Lucantonio Porzio a Francesco D’Andrea. ${ }^{25}$ Tenía que reconstituir el campo político y conceptual en el que se construye el pensamiento de Vico. El desafío era tanto más importante cuanto que el propio Vico es poco explícito sobre esta herencia que apenas evoca en su autobiografía. También sobre este punto podría desarrollarse una investigación para pensar la articulación entre las primeras Luces y las que se desarrollarán admirablemente en Nápoles en el siglo XVIII, ya se trate de Pietro Giannone, de Gaetano Filangieri, de Mario Pagano o de Vincenzo Cuoco, por no citar sino algunos ejemplos significativos. Prestar atención a las herencias recibidas por Vico implicaba también considerar con atención la herencia que él mismo deja a las Luces, así como a la historia de las ideas en Italia de manera general (Pasquale Villari, Giuseppe Ferrari). Del mismo modo, entiendo que la relación entre Vico y Gramsci es una pista fecunda por explorar. ${ }^{26}$

$\mathrm{Al}$ término de este recorrido viquiano rápidamente esbozado, creo que si tuviera que resumir las características de mi Vico, no puedo dejar de reconocer la fuerza de un autor y de una lengua imposibles de resumir en unas cuantas grandes tesis, pero capaces de suscitar siempre un cuestionamiento problemático y vivaz.

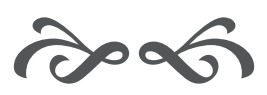

24. Véase P. GIRARD, «'Una piramide nel deserto’? Appunti sulla Scienza nuova di Vico», Bollettino del Centro di studi vichiani, XLV, 2015, pp. 43-57.

25. Es este el reto de mi «Comme des lumières jamais vues». Matérialisme et radicalité politique dans les premières Lumières à Naples (1647-1744), Champion, París, 2016. Véase también «L'invention de la modernité à Naples», P. GiRARD (Dir.), Archives de philosophie, 80, 3/2017, passim.

26. «De Vico à Gramsci. Éléments pour une confrontation», en Laboratoire italien, «Gramsci da un secolo all'altro», R. Descendre, F. Frosini (Dir.), n 18, 2016: https://laboratoireitalien.revues.org/1039 
<smiles>[As]</smiles> 